\title{
Hipotireoidismo adquirido tratado como obesidade exógena: a importância do controle do crescimento
}

\author{
Acquired hypothyroidism treated as exogen obesity: the importance of growth follow-up
}

\author{
Mariana Porto Zambon ${ }^{1}$, Maria Ângela R. G. M. Antonio', Roberto Teixeira Mendes ${ }^{1}$, Antônio de Azevedo B. Filho
}

\section{RESUMO}

Objetivo: Demonstrar a importância da interpretação do acompanhamento pôndero-estatural de crianças e adolescentes obesos.

Descrição do caso: Menina de 12 anos e 11 meses encaminhada a um ambulatório terciário para acompanhamento de obesidade e dislipidemia. Referia ganho de peso a partir de oito anos, negava fazer atividade física e possuía alimentação adequada. Relatava obesidade na família do pai. Ao exame, bom estado geral, diminuição da pilificação e mixedema generalizados, pele ressecada e áspera. Peso com percentil entre 90 e 97 , índice de massa corpórea (IMC) acima do percentil 97 e estatura abaixo do canal de crescimento. Exames laboratoriais: T4: 0,04ng/dL, TSH: >100uUI/mL, colesterol total: 326mg/dL, HDL colesterol: 34mg/dL, LDL colesterol: $45 \mathrm{mg} / \mathrm{dL}$, triglicérides: $1599 \mathrm{mg} / \mathrm{dL}$, glicemia em jejum: $81 \mathrm{mg} / \mathrm{dL}$ e hemograma com discreta anemia normocrômica e normocítica. Fez-se o diagnóstico de hipotireoidismo e introduziu-se hormônio tireoidiano com boa resposta. A paciente trouxe 23 medidas prévias de peso e estatura, mostrando comprometimento de estatura e aumento de peso não valorizado.

Comentários: A análise dos gráficos de crescimento é fundamental para o acompanhamento de todas as crianças e adolescentes, principalmente aquelas com sobrepeso e obesidade. A desaceleração da curva de crescimento em altura sugere doença associada; neste caso, o hipotireoidismo adquirido.

Palavras-chave: crescimento; hipotireoidismo; obesidade.

\section{ABSTRACT}

Objective: To show the value of using the follow-up growth charts in clinical evaluation of obese children and adolescents.

Case description: A 12 years and 11 months-old girl referred to a tertiary out-patient clinic to evaluate obesity and lipid abnormalities. She had weight gain since eight years old, had no physical activity and followed adequate eating habits. Obesity was referred in father's family. In physical examination, she looked well, with lack of body hair, generalized mixedema, dry and rough skin. Weight was between percentile 90 and 97; BMI was over the percentile 97 and height was under the growth channel. Laboratory exams: T4: $0.04 \mathrm{ng} / \mathrm{dL}$, TSH: $>100 \mathrm{uUI} / \mathrm{mL}$, total cholesterol: 326mg/dL, HDL cholesterol: $34 \mathrm{mg} / \mathrm{dL}$, LDL cholesterol: $45 \mathrm{mg} / \mathrm{dL}$, triglycerides: $1599 \mathrm{mg} / \mathrm{dL}$, fasting glicose: $81 \mathrm{mg} / \mathrm{dL}$ and hemogram with normocromic and normocytic anemia. Hypothyroidism diagnosis was made and thyroid hormone was introduced with good response. The patient brought 23 previous heights and weight measurements, which showed height drop and weight gain in the growth chart, all of them undervalued.

Comments: Growth chart analysis is important for the follow-up of children and adolescents, especially those with overweight and obesity. The linear growth slowing suggests an associated sickness; in this case, an acquired hypothyroidism.

Key-words: growth; hypothyroidism; obesity.
Instituição: Departamento de Pediatria da Faculdade de Ciências Médicas da Universidade Estadual de Campinas (FCM-Unicamp), Campinas, SP, Brasil ${ }^{1}$ Professor-assistente Doutor do Departamento de Pediatria, médico do Ambulatório de Crianças e Adolescentes Obesos do Hospital de Clínicas da Unicamp, Campinas, SP, Brasil

2Professor-associado do Departamento de Pediatria, médico do Ambulatório de Crianças e Adolescentes Obesos do Hospital de Clínicas da Unicamp, Campinas, SP, Brasil
Endereço para correspondência:

Mariana Porto Zambon

Rua Botafogo, 151/491

CEP 13130-601 - Campinas/SP

E-mail: mzambon@fcm.unicamp.br

Recebido em: 10/7/08

Aprovado em: 28/9/08 


\section{Introdução}

A medicina evoluiu de forma significativa nas últimas décadas, facilitando a realização de exames laboratoriais e de imagem. A clínica, no entanto, embasada na anamnese e no exame físico, ainda deve ser o alicerce do ato médico e ter a função de orientar as hipóteses diagnósticas e, por consequência, solicitar e interpretar exames complementares. Atualmente, observa-se, em alguns serviços, a solicitação de exames de rotina muitas vezes desnecessários, com aumento significativo nos custos. Por outro lado, alguns exames podem definir um diagnóstico independentemente de raciocínio clínico prévio.

Em Pediatria, a monitorização do crescimento pela antropometria é de suma importância na clínica diária, pois propicia uma avaliação longitudinal do paciente ${ }^{(1)}$. A observação de diminuição na velocidade de crescimento justifica sempre a investigação sistemática, com o objetivo de afastar deficiências alimentares, doenças crônicas, metabólicas e genéticas. Por outro lado, na criança com obesidade exógena e ganho ponderal aumentado, observam-se sinais de maturação precoce e respectivo aumento de estatura ${ }^{(1)}$.

A obesidade, hoje considerada endêmica, é multifatorial, sendo a maioria dos casos de natureza exógena, ou seja, secundária a uma diminuição da atividade física associada ao aumento de ingestão de alimentos ricos em gorduras e carboidratos $^{(2,3)}$. Porém, pode estar associada a outras causas, principalmente quando acompanhada de alterações identificáveis no monitoramento do crescimento.

A discussão deste caso tem como objetivo demonstrar a importância da interpretação do acompanhamento pônderoestatural e de maturação sexual de crianças e adolescentes obesos, para se identificarem doenças subjacentes ou concomitantes.

\section{Descrição do caso}

Adolescente do sexo feminino, com 12 anos e 11 meses, encaminhada para um ambulatório terciário de crianças e adolescentes obesos para acompanhar sua obesidade dislipidemia. Relata ter sido sempre "gordinha", com ganho de peso mais significativo a partir dos oito anos de idade, sem motivo desencadeante. Refere ser preguiçosa e sonolenta. Não faz atividade física e assiste TV a tarde toda. Em relação à alimentação, não toma café da manhã, mas, de acordo com suas informações, é adequada. Refere urina clara e abundante.
O hábito intestinal é de uma vez por semana. Nega menarca. Nasceu com 3.300g. Nos antecedentes patológicos, há relato de epifiolistese bilateral, corrigida cirurgicamente. Os avós paternos e os irmãos são obesos; o pai tem sobrepeso. Ao exame, encontrava-se em bom estado geral, quieta, corada, com diminuição da pilificação e mixedema generalizado. A pele estava ressecada e áspera. A tireoide era fibroelástica, sem nódulos ou cistos. O desenvolvimento puberal de Tanner era estadio M3P1. Observou-se, na consulta, peso de $67 \mathrm{~kg}$ (percentil entre 90 e 97), estatura de 1,46m (percentil entre 3 e 10, abaixo do canal de crescimento), índice de massa corpórea (IMC) de 31,43 (acima do percentil 97), pressão arterial de $100 / 70 \mathrm{mmHg}$ (normal), frequência cardíaca de 69 batimentos/minuto e frequência respiratória de 24 incursões/minuto.

A paciente trazia os seguintes exames laboratoriais: T4: 0,04ng/dL (baixo); TSH: >100uUI/mL (aumentado); colesterol total: $326 \mathrm{mg} / \mathrm{dL}$; HDL colesterol: $34 \mathrm{mg} / \mathrm{dL}$; LDL colesterol: $45 \mathrm{mg} / \mathrm{dL}$; triglicérides: $1599 \mathrm{mg} / \mathrm{dL}$; glicemia em jejum: $81 \mathrm{mg} / \mathrm{dL}$ e hemograma com discreta anemia normocrômica e normocítica (Hemoglobina: 10,90g/dL e hematócrito: $31,8 \%$ ).

$\mathrm{Na}$ vigência desses exames, foi feito o diagnóstico de hipotireoidismo, sendo solicitadas dosagens de anticorpos antitireoidiano, idade óssea e medidas antropométricas anteriores: anticorpo tireoidiano microssomal: $187 \mathrm{IU} / \mathrm{mL}$ (normal <35), anticorpo tireoidiano tireoglobulina: 188IU/ $\mathrm{mL}$ (normal <115), Idade óssea: 12 anos (Greulich-Pyle). Como medidas anteriores, a paciente trouxe 23 medidas de peso e estatura realizadas em Unidade Básica de Saúde entre abril de 1998 e março de 2007.

Confirmada a hipótese, introduziu-se hormônio tireoidiano lentamente até que se atingisse $100 \mathrm{mg} / \mathrm{dia}$, com boa resposta. Após seis meses de tratamento, a paciente apresentava-se sem queixas, com melhora da sonolência e da constipação intestinal. Ao exame, houve melhora evidente do mixedema, da pele ressecada e o retorno da pilificação normal em todo o corpo. Quanto às medidas, observou-se: peso: $54 \mathrm{~kg}$ (perda de $8 \mathrm{~kg}$, percentil entre 50 e 75); estatura: $1,48 \mathrm{~m}$ (ganho de $2 \mathrm{~cm}$, percentil entre 3 e 10); IMC: 24,65 (percentil entre 85 e 95; sobrepeso); estágio puberal de Tanner: M3P2. Nos exames laboratoriais de controle, verificou-se: T4: 1,32ng/dL (normal), TSH: $3,65 \mathrm{uUI} / \mathrm{mL}$ (normal), colesterol total: $115 \mathrm{mg} /$ dL, HDL colesterol: $27 \mathrm{mg} / \mathrm{dL}$, LDL colesterol: $56 \mathrm{mg} / \mathrm{dL}$, triglicérides: $162 \mathrm{mg} / \mathrm{dL}$. Após dez meses de tratamento, apresentou menarca. 
Este artigo foi submetido ao Comitê de Ética em Pesquisa Faculdade de Ciências Médicas da Universidade Estadual de Campinas (FCM-Unicamp) e aprovado para publicação.

\section{Discussão}

A adolescente foi encaminhada ao Ambulatório de Crianças e Adolescentes Obesos do Hospital das Clínicas da Unicamp, ambulatório terciário, interdisciplinar, que funciona há aproximadamente três anos e já atendeu mais de 150 pacientes de várias cidades da região. $\mathrm{Na}$ primeira avaliação, a menina já trazia o resultado dos exames laboratoriais sugestivos de hipotireoidismo. O que chamou a atenção da equipe médica foi o fato de tais exames terem sido solicitados de forma rotineira, visando apenas investigar a obesidade, mas sem aventar a hipótese de hipotireoidismo, obrigatória na situação clínica apresentada.

O hipotireoidismo é uma das doenças endócrinas mais frequentes na Pediatria. Resulta da deficiência de hormônios tireoidianos, que necessitam da integridade do eixo hipotálamo-hipófise-tireoide para que sejam produzidos. Os hormônios tireoidianos atuam em praticamente todos os tecidos, tendo importância significativa no crescimento e desenvolvimento da criança, por agir no metabolismo de proteínas, carboidratos e lipídeos. Em relação aos lipídeos, vale a pena ressaltar que sua falta provoca a diminuição da síntese do colesterol e sua conversão metabólica, favorecendo o aumento dos triglicérides, como observado na paciente em questão ${ }^{(4)}$.

Na clínica pediátrica, o hipotireoidismo é encontrado em duas formas distintas: o congênito e o adquirido. $\mathrm{O}$ hipotireoidismo congênito apresenta importante repercussão clínica, principalmente relacionada ao comprometimento do desenvolvimento do sistema nervoso central, o que justificou sua inclusão em testes de triagem propostos a todos os recémnascidos. Sua realização vem aumentando progressivamente em todo o mundo, inclusive na América do Sul, sendo que, no Brasil, a cobertura do programa nacional de triagem neonatal em 2005 foi de $80,2 \%{ }^{(5,6)}$.

No hipotireoidismo adquirido, o retardo mental é menos significativo, sendo as alterações de crescimento as mais afetadas $^{(4)}$. As alterações clínicas dependem do grau e da duração da falta desses hormônios, podendo ocorrer de forma lenta e progressiva, caso não seja realizado tratamento adequado. Inicialmente, há diminuição da velocidade de crescimento, cansaço, dificuldade escolar, constipação intestinal, pele e cabelo ressecados, unhas quebradiças e irregularidade do flu- xo menstrual, predominando a hipermenorreia e, em menor número, a amenorreia. Os sinais clínicos aumentam com o retardo no diagnóstico e consequente atraso na reposição hormonal, surgindo, então, o mixedema ${ }^{(4)}$.

Como visto, essa adolescente apresenta quadro clínico compatível com hipotireoidismo que, quando avaliado conjuntamente com o gráfico de crescimento, demonstra retificação da curva de estatura e ganho de peso mais significativo há aproximadamente três anos e meio, provavelmente quando a doença se iniciou. Fato relevante para os autores é que, mesmo a paciente tendo realizado consultas periódicas com medidas de peso e altura no período, tais medidas não foram devidamente analisadas e valorizadas, o que retardou o diagnóstico de hipotireoidismo, tratado de início apenas como obesidade exógena.

O diagnóstico de obesidade exógena foi feito devido à alta prevalência da doença atualmente ${ }^{(2,3)}$ e pelo fato de o IMC ser muito alto (IMC: 31,43 acima do percentil 97), o que, normalmente, não se observa em crianças com hipotireoidismo adquirido. Muitos pacientes com hipotireoidismo não apresentam ganho de peso, sendo que alguns podem até perder peso ${ }^{(7)}$. Ao se observar cuidadosamente a curva de peso, verifica-se que a partir de sete anos e meio a paciente começou a ganhar peso de forma acentuada, mantendo uma velocidade de crescimento normal até aproximadamente dez anos, deixando de crescer nos seguintes três anos e meio. Portanto, provavelmente, essa menina apresentava, inicialmente, indícios de obesidade e, após algum tempo, desenvolveu hipotireoidismo. O aumento de peso, quando ocorre no hipotireoidismo adquirido, deve-se ao acúmulo de fluído proteico no subcutâneo, conhecido como mixedema. Assim como o ganho de peso não é esperado, sua perda também não ocorre de forma sistemática e é mais frequente naqueles com quadros mais graves ${ }^{(7)}$. Neste caso, observou-se queda acentuada do peso seis meses após o uso de hormônio antireoidiano, principalmente pela redução do mixedema avaliado clinicamente.

Nos protocolos de abordagem de crianças obesas, os consensos americanos e canadenses ${ }^{(8,9)}$ preconizam uma história clínica dirigida, enfatizando antecedentes familiares, a presença de acantose nigricans, medidas de pressão arterial e circunferência abdominal e a realização de exames laboratoriais para diagnóstico de complicações cardíacas e metabólicas, destacando-se o perfil lipídico e a glicemia de jejum. Apesar de esses consensos não mencionarem a investigação de hipotireoidismo, com dosagens de T4 livre e TSH, devido à baixa incidência da doença como causa de obesidade, muitos 
serviços básicos e pediatras gerais a realizam de rotina ${ }^{(10)}$. No Brasil, Damiani ${ }^{(11)}$ discute a relevância desses exames e reforça a importância da avaliação clínica e da monitorização do crescimento, pois, clinicamente, o obeso é um maturador rápido, o que lhe confere uma aparência de criança grande e bem desenvolvida.

$\mathrm{Na}$ avaliação desse caso clínico, constata-se que a análise dos gráficos de crescimento é fundamental para o acompanhamento de todas as crianças e adolescentes, principalmente aquelas com sobrepeso e obesidade. A re- tificação da curva de crescimento sugere doença associada; neste caso, o hipotireoidismo adquirido. Este, por sua vez, isoladamente, não resulta em IMC compatível com sobrepeso e obesidade. O tratamento dessa paciente com hormônio tireoidiano mostrou melhora nos parâmetros clínicos, antropométricos, de desenvolvimento puberal e laboratorial, indicando controle do hipotireoidismo adquirido, mas mantendo diagnóstico de sobrepeso, o qual deve ser acompanhado periodicamente com orientação alimentar e de atividade física.

\section{Referências bibliográficas}

1. Zeferino AM, Barros Filho AA, Bettiol H, Barbieri MA. Monitoring growth. J Pediatr (Rio J) 2003;79(Suppl 1):S23-32.

2. World Health Organization. Obesity: preventing and managing the global epidemic: report of a WHO consultation on obesity. Geneva: World Health Organization; 1998.

3. Styne DM. Childhood and adolescent obesity. Prevalence and significance. Pediatr Clin North Am 2001;48:823-54.

4. Setian N. Hypothyroidism in children: diagnosis and treatment. J Pediatr (Rio J) 2007;83(Suppl 5):S209-16.

5. Borrajo GJ. Newborn screening in Latin America at the beginning of the $21^{\text {st }}$ century. J Inherit Metab Dis 2007;30:466-81.

6. Carvalho TM, dos Santos HP, dos Santos IC, Vargas PR, Pedrosa J. Newborn screening: a national public health programme in Brazil. $\mathrm{J}$ Inherit Metab Dis 2007;30:615.
7. Lomenick JP, El-Sayyid M, Smith WJ. Effect of levo-thyroxine treatment on weight and body mass index in children with acquired hypothyroidism. J Pediatr 2008;52:96-100

8. Barlow SE, Dietz WH. Obesity evaluation and treatment: Expert Committee recommendations. The Maternal and Child Health Bureau, Health Resources and Services Administration and the Department of Health and Human Services. Pediatrics 1998;102:e29.

9. Lau DC, Douketis JD, Morrison KM, Hramiak IM, Sharma AM, Ur E; Obesity Canada Clinical Practice Guidelines Expert Panel. 2006 Canadian clinical practice guidelines on the management and prevention of obesity in adults and children. CMAJ 2007;176:S1-13.

10. O'Brien SH, Holubkov R, Reis EC. Identification, evaluation, and management of obesity in an academic primary care center. Pediatrics 2004;14:e154-9.

11. Damiani D. Obesidade e doenças tireoidianas: mitos e realidades. Pediatria (São Paulo) 2007;29:82-3. 